\title{
Influence of Weather and Soil Parameters on Development of Wet Root Rot in Pulse Crops and Virulence Analysis of Rhizoctonia solani Isolates
}

\author{
S. C. Dubey ${ }^{1}$, Aradhika Tripathi ${ }^{1}$, B. K. Upadhyay ${ }^{1} \&$ Birendra Singh ${ }^{1}$ \\ ${ }^{1}$ Division of Plant Pathology, Indian Agricultural Research Institute, New Delhi \\ Correspondence: S. C. Dubey, Division of Plant Pathology, Indian Agricultural Research Institute, New Delhi \\ 110012, India, Tel: 91-11-25841391(H), 91-11-25848418, 25843474 (O). E-mail: scdube2002@yahoo.co.in
}

Received: July 23, 2012 Accepted: August 6, 2012 Online Published: October 12, 2012

doi:10.5539/jas.v4n11p195 URL: http://dx.doi.org/10.5539/jas.v4n11p195

\begin{abstract}
Wet root rot caused by Rhizoctonia solani Kühn in pulse crops was favored by wide range of soil parameters like temperature, humidity, $\mathrm{pH}$, electric conductivity and soil texture. The areas surveyed for the collection of the isolates showing variable atmospheric temperature and relative humidity and low to medium levels of soil organic carbon and high level of available phosphorus influenced the development of the disease incidence from $2-48 \%$. Seventy three cultivars of mungbean, twenty eight cultivars of urdbean and eight cultivars of cowpea were evaluated against virulent isolate of $R$. solani (RASC 30) to design a set of differential cultivars for virulence analysis. Two cultivars of urdbean, namely, NDU3-4 and IPU2-14, one cultivar of mungbean, namely, HUM 1 and three cultivars of cowpea, namely, V240, V585 and DCP7 showed resistant reactions. Four cultivars of urdbean, namely, TU94-2, KU323, KUG216 and B3-8-8, one cultivar of mungbean, namely, PDM54 and two cultivars of cowpea, namely, V578 and DCP13 were moderately resistant against the pathogen. The virulence analysis of 90 isolates of the pathogen representing 7 anastomosis groups (AGs) isolated from pulse crops of 16 agro-ecological regions of India on a set of differential cultivars, namely, HUM 1, PDM 54 and Pusa Vishal of mungbean, NDU 3-4, KU 323, Uttara of urdbean and V 240, V 578 and Pusa Sukomal of cowpea grouped the isolates into five pathotypes. The differential cultivar for each pathotype was identified. The pathotypes were not corresponding to the AG type of the isolates. Except one pathotype (isolate RMPG28 belonging to AG2-3), each pathotype had the isolates from different AGs.
\end{abstract}

Keywords: virulence, differential cultivars, pathotypes, Rhizoctonia solani, soil conditions

\section{Introduction}

India is recognized globally as a major pulse producing country sharing $25 \%$ of the global pulse production. India grows a variety of pulse crops under a wide range of agro-ecological conditions in $23.4 \mathrm{mh}$ with $14.6 \mathrm{mt}$ production and $625 \mathrm{~kg} \mathrm{ha}^{-1}$ productivity (Agricultural Statistics at a Glance, 2010). Biotic stresses are the major cause of low productivity of pulse crops in India. Amongst the fungal pathogens, Rhizoctonia solani Kühn [teleomorph - Thanatephorus cucumeris (Fr.) Donk] is a seed- and soil-borne plant pathogen causing web blight/wet root rot in pulse crops besides other agricultural and horticultural crops. Chickpea (Cicer arietinum $\mathrm{L}$ ), cowpea [Vigna unguiculata (L.) Walp.], mungbean [Vigna radiata (L) Wilczek], urdbean [Vigna mungo (L.) Hepper] lentil (Lens culinaris Medikus) and french bean (Phaseolus vulgaris L.) are the major pulse crops cultivated world wide under different agro-climatic conditions are affected by $R$. solani. The pathogen causes considerable yield loss in mungbean and urdbean in India (Dubey, 2003). Yield loss up to 57\% in mungbean was reported from Iran (Kaiser, 1970). The intensive crop cultivation and modified agro-practices have increased the populations of $R$. solani in soil and gradually built up new disease problems. $R$. solani is genetically diverse in respect of pathogenicity as well as cultural/morphological and physiological characters. Initially, Rhizoctonia spp. are classified in different species or groups on the basis of morphological and cultural characters (Parmeter \& Whiteny, 1970). Isolates of $R$. solani have been traditionally classified into different anastomosis groups (AGs) (Ogoshi, 1987; Carling, 1996).

Very limited studies on the virulence characterization and grouping of the host-specific isolates of $R$. solani have been carried out so far. Attempt has not been made yet to identify the pathotypes/races of $R$. solani associated 
with pulse crops based on virulence analysis on host differentials. The host differentials for pulse crops are also not available worldwide. The pathotypes of the pathogen have not been correlated with agro-ecological regions, AGs and soil and weather parameters. Keeping these points in view, the present study was aimed to standardize the host differentials to determine the pathotypes/races among the populations of $R$. solani representing various AGs associated with various pulse crops in different agro-ecological regions of India having diverse cropping sequences and to determine the influence of weather and soil parameters on disease development in different areas.

\section{Materials and Methods}

\subsection{Cultures of Rhizoctonia Solani and Cultivars of Mungbean, Urdbean and Cowpea}

Ninety (representative of 470 isolates) isolates of $R$. solani isolated from different pulse crops from 16 agro-ecological regions covering 21 states of India and being maintained in Pulse Laboratory, Division of Plant Pathology, Indian Agricultural Research Institute, New Delhi were used in the present study. The cultivars/genotypes of mungbean, urdbean and cowpea included in the present study were collected from Division of Genetics, Indian Agricultural Research Institute, New Delhi.

\subsection{Weather and Soil Parameters}

The soil samples along with diseased specimens were collected from 9 states, namely, Delhi, Uttar Pradesh, Rajasthan, Jammu and Kashmir, Uttarakhand, Panjab, Gujarat, Maharashtra and Assam. The pooled samples of each location (48 soil samples) were analyzed for nitrogen, carbon, phosphorus, salinity, $\mathrm{pH}$ and soil texture. The weather parameters as soil and atmospheric temperature, soil and atmospheric relative humidity were also recorded in these areas by using portable digital humidity and temperature recorder.

\subsection{Reaction of Different Cultivars of Mungbean, Urdbean and Cowpea against R. solani}

In order to find out resistant sources to constitute a set of differential cultivars for analysis of virulence, 28 cultivars of urdbean, 8 cultivars of cowpea and 73 cultivars of mungbean were evaluated against $R$. solani (RASC 30) under sick pot soil condition. Surface sterilized (0.1\% formalin) plastic pots (20 $\mathrm{cm} \mathrm{dia)} \mathrm{were} \mathrm{filled}$ $\left(2 \mathrm{~kg} \mathrm{pot}^{-1}\right)$ with sterilized soil (1\% formalin). The soil was inoculated 2-days prior sowing with 10-day-old inoculum (10 g kg $\mathrm{g}^{-1}$ soil) of $R$. solani multiplied on sorghum grains (Dubey et al., 2009). Ten seeds of urdbean, mungbean and cowpea in each pot were sown on August 22, 2009 seperately in three replications. The incidence of wet root rot was recorded at 15 days interval up to maturity of the crop plants.

\subsection{Virulence Analysis}

Virulence analysis of 90 representative isolates including international testers of 7 AGs of the pathogen was carried out on a set of 10 differential cultivars, namely, HUM 1, PDM 54 and Pusa Vishal of mungbean, NDU 3-4, KU 323, Uttara and KUG 216 of urdbean, and V 240, V 578 and Pusa Sukomal of cowpea in net house. Twelve seeds of each cultivar were sown on August 3, 2010 and July 13, 2011 in $20 \mathrm{~cm}$ diameter surface sterilized plastic pots $(0.1 \%$ mercuric chloride) filled with $2 \mathrm{~kg}$ sterilized soil (1\% formalin for 15 days $)$ and inoculated with 10-day old culture of $R$. solani multiplied on sorghum grains (10 $\mathrm{g} \mathrm{kg}^{-1}$ soil) 2 days before sowing. Pots with un-inoculated soils were also maintained as control for comparison. The incidence of wet root rot was recorded at 15 days interval up to maturity of the crop plants.

\subsection{Virulence Analysis of AG Groups and Their Combinations}

A set of pot experiment was conducted to test the virulence of the isolates representing two AG groups for AG1isolate RUPM83 and RUPU82 and for AG4- isolate RUPM66 and RUPU69) individually and in combinations along with hybrid culture of the same group in three replications. Surface sterilized $(0.1 \%$ formalin) plastic pots $\left(10 \mathrm{~cm}\right.$ diameter) were filled $\left(500 \mathrm{~g} \mathrm{pot}^{-1}\right)$ with sterilized soil $(1 \%$ formalin). The soil was inoculated 2 day prior sowing with 10-day-old inoculum as mentioned earlier. Ten seeds of mungbean (cv Ratna) were sown on August 1, 2009 in each pot. The incidence of wet root rot was recorded at 15 days interval up to maturity of the crop plants.

\subsection{Observations Recorded and Data Analysis}

The wet root rot incidence was recorded on the basis of number of plants showing wet root rot symptoms out of the total plants and the cultivars/genotypes were categorized into resistant ( $0-10 \%$ root rot incidence), moderate resistant ( $>10-20 \%$ root rot incidence), susceptible ( $>20-50 \%$ wet root rot incidence) and highly susceptible ( $>50 \%$ root rot incidence). Finally, the reactions were presented as resistant $(0-20 \%)$ and susceptible $(>20 \%)$ for pathotype grouping (Dubey, 2003). 


\section{Results}

\subsection{Influence of Weather and Soil Parameters}

The soil and atmospheric temperature and relative humidity recorded in different parts of the country were variable. The results (Table 1) indicated that the disease favoured by wide range of soil $\left(18.1-41.0^{\circ} \mathrm{C}\right)$ and atmospheric $\left(8.1-46.3^{\circ} \mathrm{C}\right)$ temperature and soil $(28-68 \%)$ and atmospheric $(20-87 \%)$ relative humidity. The disease development was also favoured by variable soil parameters. The soil $\mathrm{pH}$ range from 5.56-8.71 and electric conductivities from $0.03-0.51 \mathrm{ds} \mathrm{m}^{-1}$ favoured the disease development. The majority of areas surveyed showed low to medium level of soil organic carbon and total nitrogen ranging from 62.7-492.8 $\mathrm{kg} \mathrm{ha}^{-1}$. Available phosphorus was from 1.2-98.3 $\mathrm{kg} \mathrm{ha}^{-1}$ and the soil samples collected from most of the areas showed high phosphorus status. Soil texture was also variable in areas surveyed from loam to sandy loam and silty to clay loam (Table 2).

Table 1. Soil and atmospheric temperature and humidity recorded during survey in different parts of the country

\begin{tabular}{|c|c|c|c|c|c|}
\hline \multirow[b]{2}{*}{ State/District } & \multicolumn{2}{|c|}{ Soil parameters } & \multicolumn{2}{|c|}{ Atmospheric parameters } & \multirow[b]{2}{*}{ Mean range of disease incidence (\%) } \\
\hline & Temperature $\left({ }^{\circ} \mathrm{C}\right)$ & Humidity (\%) & $\begin{array}{c}\text { Temperature }\left({ }^{\circ} \mathrm{C}\right) \\
\text { Min - Max }\end{array}$ & $\begin{array}{l}\text { Relative humidity (\%) } \\
\text { Min - Max }\end{array}$ & \\
\hline \multicolumn{6}{|c|}{ Uttar Pradesh } \\
\hline Mirzapur & 19.4 & 59 & $20.3-29.3$ & $38-72$ & $15-29$ \\
\hline Mirzapur & 21.6 & 58 & $21.8-28.9$ & $40-74$ & $10-25$ \\
\hline Mirzapur & 19.2 & 62 & $20.3-29.3$ & $35-72$ & $12-20$ \\
\hline Mirzapur & 21.6 & 58 & $21.8-28.9$ & $40-74$ & $11-25$ \\
\hline Mirzapur & 20.2 & 68 & $22.1-28.5$ & $38-74$ & $12-26$ \\
\hline Jhansi & 36.1 & 52 & $34.1-40.3$ & $32-70$ & $10-36$ \\
\hline Jhansi & 35.2 & 52 & $34.1-40.3$ & $35-72$ & $8-30$ \\
\hline Jhansi & 37.5 & 56 & $32.1-41.3$ & $32-68$ & $8-40$ \\
\hline Jhansi & 36.0 & 52 & $31.1-40.3$ & $32-73$ & $14-34$ \\
\hline Gorakhpur & 21.2 & 51 & $17.8-28.0$ & $40-78$ & $5-30$ \\
\hline Gorakhpur & 18.1 & 50 & $09.2-20.8$ & $36-76$ & $7-25$ \\
\hline Gorakhpur & 18.4 & 50 & $08.1-25.8$ & $36-76$ & $10-32$ \\
\hline Kanpur & 22.4 & 58 & $17.9-28.4$ & $38-76$ & $7-36$ \\
\hline Kanpur & 21.8 & 54 & $18.1-28.2$ & $36-72$ & $7-40$ \\
\hline Varanasi & 21.8 & 56 & $20.6-25.0$ & $40-82$ & $8-23$ \\
\hline Varanasi & 19.1 & 65 & $21.6-28.0$ & $36-76$ & $10-19$ \\
\hline \multicolumn{6}{|c|}{ Rajasthan } \\
\hline Jaipur & 34.1 & 48 & $32.1-36.4$ & $30-68$ & $5-26$ \\
\hline Jaipur & 35.8 & 46 & $32.1-36.4$ & $30-68$ & $9-30$ \\
\hline Hanumangarh & 32.8 & 41 & $31.1-38.4$ & $35-62$ & $5-20$ \\
\hline \multicolumn{6}{|c|}{ Jammu \& Kashmir } \\
\hline Samba & 38.2 & 28 & $36.2-46.3$ & $26-50$ & $5-20$ \\
\hline Samba & 38.6 & 28 & $36.2-46.3$ & $26-50$ & $7-30$ \\
\hline Samba & 38.8 & 28 & $36.2-46.3$ & $26-50$ & $3-25$ \\
\hline Udhampur & 35.5 & 20 & $34.4-42.6$ & $20-48$ & $2-20$ \\
\hline \multicolumn{6}{|c|}{ Uttarakhand } \\
\hline U. S. Nagar & 32.8 & 59 & $30.2-36.8$ & $62-81$ & $5-40$ \\
\hline U.S. Nagar & 31.3 & 62 & $31.2-38.1$ & $62-72$ & $10-45$ \\
\hline U. S. Nagar & 31.1 & 62 & $31.2-38.1$ & $62-72$ & $6-48$ \\
\hline Tehri Garhwal & 29.8 & 52 & $28.9-33.8$ & $45-68$ & $5-28$ \\
\hline Tehri Garhwal & 33.8 & 60 & $29.5-35.2$ & $42-62$ & $6-35$ \\
\hline \multicolumn{6}{|c|}{ Panjab } \\
\hline Ludhiana & 32.3 & 59 & $31.2-33.8$ & $62-87$ & $7-30$ \\
\hline Ludhiana & 32.3 & 59 & $31.2-33.8$ & $62-87$ & $4-24$ \\
\hline Ludhiana & 22.3 & 59 & $31.2-33.8$ & $62-87$ & $5-25$ \\
\hline Ludhiana & 32.8 & 60 & $31.2-33.8$ & $62-87$ & $6-26$ \\
\hline \multicolumn{6}{|c|}{ Gujarat } \\
\hline Ahmedabad & 41.0 & 56 & $30.3-41.0$ & $28-70$ & $5-14$ \\
\hline Ahmedabad & 40.4 & 53 & $30.3-41.0$ & $28-70$ & $6-16$ \\
\hline Anand & 35.4 & 56 & $28.4-44.7$ & $28-70$ & $5-22$ \\
\hline Anand & 35.4 & 56 & $28.4-44.7$ & $28-70$ & $6-20$ \\
\hline Anand & 34.2 & 50 & $28.0-41.0$ & $28-70$ & $5-23$ \\
\hline Anand & 35.5 & 52 & $\begin{array}{l}28.4-44.7 \\
\text { Maharashtr }\end{array}$ & $28-70$ & $5-19$ \\
\hline Pune & 26.1 & 48 & $28.2-43.5$ & $31-63$ & $5-10$ \\
\hline Pune & 26.3 & 48 & $28.2-43.5$ & $31-63$ & $4-8$ \\
\hline \multicolumn{6}{|c|}{ Assam } \\
\hline Nalbari & 31.9 & 52 & $27.2-35.8$ & $48-74$ & $4-22$ \\
\hline Jorhat & 33.1 & 56 & $31.8-37.6$ & $52-79$ & $4-37$ \\
\hline
\end{tabular}


Table 2 Soil parameters of various soil samples collected from different parts of the India

\begin{tabular}{|c|c|c|c|c|c|c|c|}
\hline State /District & $\begin{array}{l}\text { Sample } \\
\text { No. }\end{array}$ & $\mathrm{pH}$ & $\begin{array}{c}\mathrm{EC} \\
(\mathrm{ds} / \mathrm{m})\end{array}$ & $\begin{array}{c}\text { Organic Carbon } \\
(\mathrm{g} / \mathrm{kg})\end{array}$ & $\begin{array}{c}\text { Total Nitrogen } \\
(\mathrm{kg} / \mathrm{ha})\end{array}$ & $\begin{array}{c}\text { Available P } \\
(\mathrm{kg} / \mathrm{ha})\end{array}$ & Texture Class \\
\hline \multicolumn{8}{|c|}{ Uttar Pradesh } \\
\hline Mirzapur & SPL-1 & 5.85 & 0.24 & 5.3 & $313.6(3.8: 1)^{*}$ & 94.3 & Loam \\
\hline Mirzapur & SPL-2 & 6.54 & 0.03 & 4.1 & $246.4(3.7: 1)$ & 98.3 & Loam \\
\hline Mirzapur & SPL-27 & 7.42 & 0.18 & 6.1 & $246.4(5.5: 1)$ & 82.3 & Silty clay \\
\hline Mirzapur & SPL-28 & 7.49 & 0.07 & 8.6 & $246.4(7.8: 1)$ & 81.6 & Sandy loam \\
\hline Mirzapur & SPL-29 & 8.23 & 0.10 & 5.7 & $188.1(7.1: 1)$ & 20.5 & Loam \\
\hline Jhansi & SPL-12 & 7.62 & 0.06 & 4.7 & $125.4(9.4: 1)$ & 7.3 & $\begin{array}{c}\text { Sandy clay } \\
\text { loam }\end{array}$ \\
\hline Jhansi & SPL-13 & 7.53 & 0.03 & 4.6 & $246.4(4.2: 1)$ & 90.2 & $\begin{array}{l}\text { Sandy clay } \\
\text { loam }\end{array}$ \\
\hline Jhansi & SPL-14 & 7.34 & 0.12 & 4.3 & $188.1(5.4: 1)$ & 22.5 & Sandy loam \\
\hline Jhansi & SPL-15 & 7.63 & 0.10 & 10.7 & $246.4(9.1: 1)$ & 88.7 & $\begin{array}{l}\text { Sandy clay } \\
\text { loam }\end{array}$ \\
\hline Gorakhpur & SPL-42 & 5.60 & 0.42 & 4.3 & $188.1(5.4: 1)$ & 23.5 & Loam \\
\hline Gorakhpur & SPL-43 & 8.05 & 0.14 & 3.9 & $188.1(4.9: 1)$ & 25.6 & Sandy loam \\
\hline Gorakhpur & SPL-44 & 5.56 & 0.21 & 3.6 & $156.8(5.1: 1)$ & 20.4 & Loam \\
\hline Varanasi & SPL-30 & 8.24 & 0.21 & 5.3 & $188.1(7.1: 1)$ & 37.1 & Clay loam \\
\hline Varanasi & SPL-46 & 7.93 & 0.20 & 5.7 & $246.4(5.2: 1)$ & 39.6 & Loam \\
\hline Kanpur & SPL-40 & 6.69 & 0.20 & 4.7 & $188.1(5.9: 1)$ & 20.3 & Loam \\
\hline Kanpur & SPL-41 & 7.75 & 0.15 & 5.0 & $246.4(4.5: 1)$ & 21.0 & Loam \\
\hline Jaunpur & SPL-45 & 6.62 & 0.11 & 3.1 & $125.4(6.2: 1)$ & 1.2 & Loam \\
\hline \multicolumn{8}{|c|}{ Rajsthan } \\
\hline Jaipur & SPL-3 & 7.61 & 0.03 & 1.8 & $188.1(2.3: 1)$ & 29.9 & Sandy loam \\
\hline Jaipur & SPL-4 & 7.69 & 0.16 & 1.5 & $125.4(3.0: 1)$ & 32.0 & Sandy loam \\
\hline Hanumangarh & SPL-5 & 8.07 & 0.04 & 1.7 & $125.4(3.4: 1)$ & 19.2 & Loamy sand \\
\hline Sriganganager & SPL-6 & 7.99 & 0.08 & 2.6 & $156.8(3.7: 1)$ & 34.1 & Loamy sand \\
\hline \multicolumn{8}{|c|}{ Jammu \& Kashmir } \\
\hline Samba & SPL-7 & 7.44 & 0.51 & 3.1 & $188.1(3.9: 1)$ & 20.9 & Sandy loam \\
\hline Samba & SPL-8 & 8.25 & 0.25 & 6.2 & $313.6(4.4: 1)$ & 24.4 & Sandy loam \\
\hline Samba & SPL-9 & 8.09 & 0.07 & 2.4 & $125.4(4.8: 1)$ & 19.6 & Loam \\
\hline Udhampur & SPL-10 & 7.98 & 0.19 & 4.3 & $125.4(8.6: 1)$ & 39.2 & Clay loam \\
\hline \multicolumn{8}{|c|}{ Uttarakhand } \\
\hline U. S. Nagar & SPL-16 & 5.64 & 0.15 & 12.7 & $313.6(8.6: 1)$ & 64.0 & $\begin{array}{c}\text { Sandy clay } \\
\text { loam }\end{array}$ \\
\hline U. S. Nagar & SPL-17 & 6.62 & 0.10 & 17.2 & $492.8(7.7: 1)$ & 52.3 & $\begin{array}{l}\text { Sandy clay } \\
\text { loam }\end{array}$ \\
\hline U. S. Nagar & SPL-18 & 6.26 & 0.06 & 10.2 & $250.8(9.1: 1)$ & 42.6 & $\begin{array}{l}\text { Sandy clay } \\
\text { loam }\end{array}$ \\
\hline Tehri Garhwal & SPL-19 & 6.05 & 0.10 & 8.9 & $282.2(7.4: 1)$ & 58.9 & Silt loam \\
\hline Tehri Garhwal & SPL-20 & 6.07 & 0.13 & 24.9 & $313.6(17.1: 1)$ & 40.3 & Silt loam \\
\hline Tehri Garhwal & SPL-21 & 5.86 & 0.08 & 8.9 & $246.4(8.1: 1)$ & 55.7 & Silt loam \\
\hline \multicolumn{8}{|c|}{ Panjab } \\
\hline Ludhiana & SPL-22 & 7.69 & 0.07 & 6.7 & $188.1(8.4: 1)$ & 34.1 & $\begin{array}{l}\text { Sandy clay } \\
\text { loam }\end{array}$ \\
\hline Ludhiana & SPL-23 & 7.82 & 0.20 & 5.4 & $188.1(6.7: 1)$ & 32.6 & $\begin{array}{l}\text { Sandy clay } \\
\text { loam }\end{array}$ \\
\hline Ludhiana & SPL-24 & 8.28 & 0.05 & 9.4 & $188.1(11.7: 1)$ & 30.2 & $\begin{array}{l}\text { Sandy clay } \\
\text { loam }\end{array}$ \\
\hline Ludhiana & SPL-25 & 7.81 & 0.09 & 2.9 & $125.4(5.8: 1)$ & 26.4 & Sandy loam \\
\hline Ludhiana & SPL-26 & 7.52 & 0.13 & 7.3 & $246.4(6.6: 1)$ & 25.0 & Sandy loam \\
\hline \multicolumn{8}{|c|}{ Gujarat } \\
\hline Ahmedabad & SPL-31 & 8.60 & 0.07 & 2.8 & $62.7(14.0: 1)$ & 16.6 & Loamy sand \\
\hline Ahmedabad & SPL-32 & 8.67 & 0.03 & 2.6 & $94.0(6.5: 1)$ & 17.0 & Loamy sand \\
\hline Anand & SPL-33 & 8.66 & 0.13 & 2.9 & $94.0(7.2: 1)$ & 81.0 & Clay loam \\
\hline Anand & SPL-34 & 8.71 & 0.09 & 2.8 & $125.4(5.6: 1)$ & 75.0 & Clay loam \\
\hline Anand & SPL-35 & 8.55 & 0.05 & 1.9 & $125.4(3.8: 1)$ & 64.2 & Clay loam \\
\hline Anand & SPL-36 & 6.80 & 0.27 & $\begin{array}{l}1.9 \\
\text { Maharasl }\end{array}$ & $125.4(3.8: 1)$ & 60.3 & Clay loam \\
\hline Pune & SPL-37 & 8.45 & 0.14 & 6.0 & $219.5(6.6: 1)$ & 35.4 & Clay loam \\
\hline Pune & SPL-38 & 8.46 & 0.08 & 6.6 & $282.2(5.5: 1)$ & 37.0 & Clay loam \\
\hline Pune & SPL-39 & 8.19 & 0.10 & 6.7 & $188.1(8.4: 1)$ & 24.4 & Clay loam \\
\hline \multicolumn{8}{|c|}{ Assam } \\
\hline Nalbari & SPL-47 & 5.83 & 0.11 & 8.5 & $313.6(6.1: 1)$ & 25.2 & Sandy loam \\
\hline Jorhat & SPL-48 & 6.34 & 0.15 & 5.6 & $219.5(6.2: 1)$ & 22.2 & Sandy loam \\
\hline
\end{tabular}

Low carbon- $5 \mathrm{~g} / \mathrm{kg}$, medium carbon- $>5-7.5 \mathrm{~g} / \mathrm{kg}$ and high carbon- $>7.5 \mathrm{~g} / \mathrm{kg}$;

Low phosphorus- $11 \mathrm{~kg} / \mathrm{ha}$, medium phosphorus- $>11-25 \mathrm{~kg} / \mathrm{ha}$ and high phosphorus- $>25 \mathrm{~kg} / \mathrm{ha}$;

*Figures given in parentheses are $\mathrm{C}: \mathrm{N}$ rations. 


\subsection{Reaction of Different Cultivars}

The results (Table 3 ) indicated that out of 28 cultivars of urdbean, only 2 cultivars, namely, NDU 3-4 and IPU 2-14, out of 73 cultivars of mungbean, one cultivar, namely, HUM 1 and out of 8 cultivars of cowpea, 3 cultivars, namely, V 240, V 585 and DCP 7 showed resistant reaction. Four cultivars of urdbean, namely, TU 94-2, KU 323, KUG 216, B 3-8-8, one cultivar of mungbean, namely, PDM 54 and 2 cultivars of cowpea, namely, V 578 and DCP 13 were found moderately resistant against the disease. Rest 96 cultivars of urdbean, mungbean and cowpea showed susceptible to highly susceptible reactions.

Table 3. Screening of different varieties of urdbean, mungbean and cowpea against Rhizoctonia solani (RASC 30)

\begin{tabular}{|c|c|c|c|c|}
\hline \multirow{2}{*}{ Crop/Varieties } & \multicolumn{2}{|c|}{ Mortality (\%) } & \multirow{2}{*}{ Total mortality (\%) } & \multirow{2}{*}{ Reaction } \\
\hline & Pre emergence $(\%)$ & Post emergence (\%) & & \\
\hline \multicolumn{5}{|c|}{ Urdbean } \\
\hline Azad urd & 26.67 & 0.00 & 26.67 & $\mathrm{~S}$ \\
\hline В 3-8-8 & 20.00 & 0.00 & 20.00 & MR \\
\hline Ballabh urd-1 & 16.67 & 6.67 & 23.34 & $\mathrm{~S}$ \\
\hline Barabanki local & 20.00 & 6.67 & 26.67 & $\mathrm{~S}$ \\
\hline BS 23-5 & 53.33 & 0.00 & 53.33 & HS \\
\hline IPU 2-14 & 0.00 & 0.00 & 0.00 & $\mathrm{R}$ \\
\hline IPU 2-33 & 63.00 & 3.33 & 66.33 & HS \\
\hline IPU 5-13 & 33.33 & 0.00 & 33.33 & $\mathrm{~S}$ \\
\hline KU 323 & 13.33 & 3.33 & 16.66 & MR \\
\hline KU 99-4 & 33.33 & 3.33 & 36.66 & $\mathrm{~S}$ \\
\hline KU 99-5 & 36.67 & 0.00 & 36.67 & S \\
\hline KU 99-20 & 40.00 & 0.00 & 40.00 & S \\
\hline KU 99- 21 & 40.00 & 10.00 & 50.00 & $\mathrm{~S}$ \\
\hline KUG 6-3 & 63.33 & 3.33 & 66.66 & HS \\
\hline KUG 216 & 13.33 & 0.00 & 13.33 & MR \\
\hline Mash 1-1 & 26.67 & 3.33 & 30.00 & $\mathrm{~S}$ \\
\hline MBU 108 & 26.67 & 3.33 & 30.00 & $\mathrm{~S}$ \\
\hline NDU 3-4 & 10.00 & 0.00 & 10.00 & $\mathrm{R}$ \\
\hline NDU 3-5 & 26.67 & 13.33 & 40.00 & $\mathrm{~S}$ \\
\hline NDU 5-3 & 93.33 & 0.00 & 93.33 & HS \\
\hline MDU 5-7 & 26.67 & 0.00 & 26.67 & $\mathrm{~S}$ \\
\hline OBG 31 & 73.33 & 3.33 & 76.66 & HS \\
\hline Pant U-31 & 46.67 & 0.00 & 46.67 & $\mathrm{~S}$ \\
\hline Sarla & 46.67 & 0.00 & 46.67 & $\mathrm{~S}$ \\
\hline TU 94-2 & 20.00 & 0.00 & 20.00 & MR \\
\hline Uttara & 33.33 & 0.00 & 33.33 & $\mathrm{~S}$ \\
\hline UH 4-6 & 50.00 & 3.33 & 53.33 & HS \\
\hline VallabhUrd1 & 40.00 & 3.33 & 43.33 & $\mathrm{~S}$ \\
\hline \multicolumn{5}{|c|}{ Mungbean } \\
\hline AKM 99-10 & 23.33 & 6.67 & 30.00 & S \\
\hline AKM 99-14 & 50.00 & 0.00 & 50.00 & S \\
\hline Barabanki & 20.00 & 30.00 & 50.00 & S \\
\hline BDU 1 & 35.00 & 5.00 & 40.00 & $\mathrm{~S}$ \\
\hline C 5 & 70.00 & 10.00 & 80.00 & HS \\
\hline C 6 & 50.00 & 5.00 & 55.00 & HS \\
\hline CGG 973 & 35.00 & 40.00 & 75.00 & HS \\
\hline COGG 912 & 43.33 & 16.67 & 60.00 & HS \\
\hline Ganga 8 & 55.00 & 15.00 & 70.00 & HS \\
\hline GM 4-2 & 35.00 & 30.00 & 65.00 & HS \\
\hline GM 5-8 & 50.00 & 20.00 & 70.00 & HS \\
\hline HUM-1 & 10.00 & 0.00 & 10.00 & $\mathrm{R}$ \\
\hline IPM 2-9-1 & 45.00 & 35.00 & 80.00 & HS \\
\hline IPM 2-9-3 & 30.00 & 5.00 & 35.00 & $\mathrm{~S}$ \\
\hline IPU 2-33 & 30.00 & 15.00 & 45.00 & $\mathrm{~S}$ \\
\hline IPU 7-19 & 20.00 & 40.00 & 60.00 & HS \\
\hline IPU 7-3 & 25.00 & 40.00 & 65.00 & HS \\
\hline K 851 & 36.67 & 10.00 & 46.67 & $\mathrm{~S}$ \\
\hline KM 2268 & 30.00 & 40.00 & 70.00 & HS \\
\hline KM 2272 & 30.00 & 15.00 & 45.00 & $\mathrm{~S}$ \\
\hline
\end{tabular}




\begin{tabular}{|c|c|c|c|c|}
\hline Kopargaon & 45.00 & 5.00 & 50.00 & $\mathrm{~S}$ \\
\hline KU 99-19 & 60.00 & 10.00 & 70.00 & HS \\
\hline KUG 531 & 40.00 & 0.00 & 40.00 & $\mathrm{~S}$ \\
\hline LGG 946 & 35.00 & 20.00 & 55.00 & HS \\
\hline Meha & 33.33 & 0.00 & 33.33 & $\mathrm{~S}$ \\
\hline MGG 359 & 40.00 & 0.00 & 40.00 & $\mathrm{~S}$ \\
\hline MGG 360 & 35.00 & 30.00 & 65.00 & HS \\
\hline MH 2-15 & 50.00 & 0.00 & 50.00 & $\mathrm{~S}$ \\
\hline MH 709 & 75.00 & 5.00 & 80.00 & HS \\
\hline MH 721 & 40.00 & 0.00 & 40.00 & $\mathrm{~S}$ \\
\hline MH 729 & 40.00 & 30.00 & 70.00 & HS \\
\hline ML 1299 & 30.00 & 35.00 & 65.00 & HS \\
\hline ML 131 & 26.67 & 16.67 & 43.34 & $\mathrm{~S}$ \\
\hline ML 1354 & 65.00 & 10.00 & 75.00 & HS \\
\hline ML 1472 & 55.00 & 15.00 & 70.00 & HS \\
\hline ML 5 & 33.33 & 13.33 & 46.66 & $\mathrm{~S}$ \\
\hline ML 818 & 40.00 & 16.67 & 56.67 & HS \\
\hline NDM 7-33 & 40.00 & 5.00 & 45.00 & $\mathrm{~S}$ \\
\hline NDM 9-18 & 45.00 & 25.00 & 70.00 & HS \\
\hline NDU 9-15 & 55.00 & 0.00 & 55.00 & HS \\
\hline OGG 56 & 30.00 & 3.33 & 33.33 & $\mathrm{~S}$ \\
\hline Pant M 4 & 63.33 & 13.33 & 76.66 & HS \\
\hline Pant M 5 & 40.00 & 13.33 & 53.33 & HS \\
\hline PDM 11 & 60.00 & 0.00 & 60.00 & HS \\
\hline PDM 54 & 13.33 & 6.67 & 20.00 & MR \\
\hline PS 16 & 30.00 & 5.00 & 35.00 & $\mathrm{~S}$ \\
\hline PU 31 & 45.00 & 0.00 & 45.00 & $\mathrm{~S}$ \\
\hline Pant U 30 & 15.00 & 40.00 & 55.00 & HS \\
\hline Pusa 2072 & 26.67 & 3.33 & 30.00 & $\mathrm{~S}$ \\
\hline Pusa 9531 & 56.67 & 10.00 & 66.67 & HS \\
\hline Pusa 971 & 70.00 & 0.00 & 70.00 & HS \\
\hline Pusa 972 & 33.33 & 33.33 & 66.66 & HS \\
\hline Pusa Baisakhi & 65.00 & 15.00 & 80.00 & HS \\
\hline Pusa Vishal & 65.00 & 0.00 & 65.00 & HS \\
\hline Ratna & 43.33 & 20.00 & 63.33 & HS \\
\hline RMG 977 & 15.00 & 50.00 & 65.00 & HS \\
\hline RMG 987 & 0.00 & 70.00 & 70.00 & HS \\
\hline RMG 989 & 25.00 & 15.00 & 40.00 & $\mathrm{~S}$ \\
\hline RUG 1 & 30.00 & 10.00 & 40.00 & S \\
\hline RVSM 11 & 0.00 & 45.00 & 45.00 & $\mathrm{~S}$ \\
\hline Samrat & 20.00 & 25.00 & 45.00 & $\mathrm{~S}$ \\
\hline Satya & 60.00 & 0.00 & 60.00 & HS \\
\hline SB 25-19 & 40.00 & 30.00 & 70.00 & HS \\
\hline SG 33-5 & 70.00 & 3.33 & 73.33 & HS \\
\hline SG 63-14 & 85.00 & 0.00 & 85.00 & HS \\
\hline SGC 16 & 45.00 & 10.00 & 55.00 & HS \\
\hline TARM 1 & 73.33 & 3.33 & 76.66 & HS \\
\hline TARM 18 & 26.67 & 13.33 & 40.00 & $\mathrm{~S}$ \\
\hline TJM 15 & 55.00 & 10.00 & 65.00 & HS \\
\hline TMB 26 & 30.00 & 55.00 & 85.00 & HS \\
\hline UH 4-4 & 75.00 & 0.00 & 75.00 & HS \\
\hline VBG 4-31 & 15.00 & 50.00 & 65.00 & HS \\
\hline VBG 4-8 & 55.00 & 0.00 & 55.00 & HS \\
\hline \multicolumn{5}{|c|}{ Cowpea } \\
\hline BM 30 & 40.00 & 0.00 & 40.00 & $\mathrm{~S}$ \\
\hline DCP 13 & 13.33 & 0.00 & 13.33 & MR \\
\hline DCP 7 & 6.67 & 3.33 & 10.00 & $\mathrm{R}$ \\
\hline Pusa Sukomal & 33.33 & 0.00 & 33.33 & $\mathrm{~S}$ \\
\hline V 130 & 13.33 & 26.67 & 40.00 & S \\
\hline V 240 & 0.00 & 0.00 & 0.00 & $\mathrm{R}$ \\
\hline V 578 & 6.67 & 13.33 & 20.00 & MR \\
\hline V 585 & 6.67 & 0.00 & 6.67 & $\mathrm{R}$ \\
\hline
\end{tabular}

Note: R (resistant) $=0-10 \%$ mortality, MR (moderate resistant) $=>10-20 \%$, S (susceptible) $=>20-50 \%$ and HS (highly susceptible) $=>50 \%$ mortality. 


\subsection{Virulence Analysis of AG Groups and Their Combinations}

There was no significant difference was observed in the disease incidence recorded (30-35\%) in the pots inoculated with different AGs, combinations of AGs and their hybrid.

\subsection{Virulence Analysis}

The results (Table 4) indicated that out of 90 isolates, 77 isolates caused moderately susceptible to susceptible reactions on a set of 10 differential cultivars. Only 13 isolates caused resistant reaction on the cultivars evaluated (Table 4). Based on the virulence on a set of the cultivars, the isolates were grouped into 5 pathotypes. The urdbean cultivar KU323 showed resistant reaction against two isolates, namely, RMHG24 (AG1) and RUPP93 (AG5) and considered as differential for the first group. The cultivar Uttara considered differential for the fourth group showing resistant reaction against four isolates, namely, RAPS3 (AG1), RMHM6 (AG5), RUPG103 (AG5) and RS9 (AG3). The urdbean cultivar KUG 216 considered differential for the third group consisting of 6 isolates, namely, RGJC18 (AG2-2), RKLC1 (AG2-3), RKLC 4 (UD), RMHM3 (AG2-3), RPBU5 (AG4) and RRJG3 (UD) showing resistant reaction. The fourth group had only one isolate RGJG 4 (UD) differentiated by cowpea cultivar V 578 showing resistant reaction. The fifth group had most of the isolates (77) showing susceptible to highly susceptible reactions against 10 cultivars used for virulence analysis (Table 5). The pathotypes were not corresponding to the AG type of the isolates.

Table 4. Reaction of mungbean, urdbean and cowpea cultivars against different isolates of $R$. solani during 2009-10 and 2010-11

\begin{tabular}{|c|c|c|c|c|c|c|c|c|c|c|c|}
\hline \multirow{3}{*}{ Isolates } & \multirow{3}{*}{ AG type } & \multicolumn{10}{|c|}{ Disease incidence (\%) } \\
\hline & & \multicolumn{3}{|c|}{ Mungbean } & \multicolumn{4}{|c|}{ Urdbean } & \multicolumn{3}{|c|}{ Cowpea } \\
\hline & & Hum 1 & PDM 54 & Pusa Vishal & NDU 34 & KU 323 & Uttara & KUG 216 & V 240 & V 578 & Pusa Sukomal \\
\hline RAPG 12 & AG5 & $\mathrm{S}$ & $\mathrm{S}$ & $\mathrm{S}$ & $\mathrm{S}$ & $\mathrm{S}$ & $\mathrm{S}$ & $\mathrm{S}$ & $\mathrm{S}$ & $\mathrm{S}$ & $\mathrm{S}$ \\
\hline RAPG 14 & AG2-3 & $\mathrm{S}$ & $\mathrm{S}$ & $\mathrm{S}$ & $\mathrm{S}$ & $\mathrm{S}$ & $\mathrm{S}$ & $\mathrm{S}$ & $\mathrm{S}$ & $\mathrm{S}$ & $\mathrm{S}$ \\
\hline RAPS3 & AG1 & $\mathrm{S}$ & $\mathrm{S}$ & $\mathrm{S}$ & $\mathrm{S}$ & $\mathrm{S}$ & $\mathrm{R}$ & $\mathrm{S}$ & $\mathrm{S}$ & $\mathrm{S}$ & $\mathrm{S}$ \\
\hline RASC5 & AG2-2 & $\mathrm{S}$ & $\mathrm{S}$ & $\mathrm{S}$ & S & $\mathrm{S}$ & S & $\mathrm{S}$ & $\mathrm{S}$ & S & $\mathrm{S}$ \\
\hline RASC8 & AG2-2 & $\mathrm{S}$ & $\mathrm{S}$ & $\mathrm{S}$ & $\mathrm{S}$ & $\mathrm{S}$ & $\mathrm{S}$ & $\mathrm{S}$ & $\mathrm{S}$ & $\mathrm{S}$ & $\mathrm{S}$ \\
\hline RASC 26 & AG4 & S & S & $\mathrm{S}$ & S & S & S & S & S & S & S \\
\hline RASC 27 & AG2-3 & $\mathrm{S}$ & $\mathrm{S}$ & $\mathrm{S}$ & $\mathrm{S}$ & $\mathrm{S}$ & $\mathrm{S}$ & $\mathrm{S}$ & $\mathrm{S}$ & $\mathrm{S}$ & $\mathrm{S}$ \\
\hline $\mathrm{RBRC1}$ & AG3 & S & S & S & S & $\mathrm{S}$ & S & $\mathrm{S}$ & S & S & S \\
\hline RCGM1 & AG3 & $\mathrm{S}$ & $\mathrm{S}$ & $\mathrm{S}$ & $\mathrm{S}$ & $\mathrm{S}$ & $\mathrm{S}$ & $\mathrm{S}$ & $\mathrm{S}$ & $\mathrm{S}$ & $\mathrm{S}$ \\
\hline RDLM1 & AG3 & $\mathrm{S}$ & $\mathrm{S}$ & $\mathrm{S}$ & $\mathrm{S}$ & $\mathrm{S}$ & $\mathrm{S}$ & $\mathrm{S}$ & $\mathrm{S}$ & $\mathrm{S}$ & $\mathrm{S}$ \\
\hline RDLM6 & AG3 & S & S & S & S & $\mathrm{S}$ & S & $\mathrm{S}$ & S & S & S \\
\hline RDLG3 & AG3 & S & S & S & S & S & S & S & S & S & S \\
\hline RGJC 18 & AG2-2 & $\mathrm{S}$ & $\mathrm{S}$ & $\mathrm{S}$ & $\mathrm{S}$ & $\mathrm{S}$ & $\mathrm{S}$ & $\mathrm{R}$ & $\mathrm{S}$ & $\mathrm{S}$ & $\mathrm{S}$ \\
\hline RGJM 24 & AG2-3 & $\mathrm{S}$ & $\mathrm{S}$ & $\mathrm{S}$ & $\mathrm{S}$ & $\mathrm{S}$ & $\mathrm{S}$ & $\mathrm{S}$ & $\mathrm{S}$ & $\mathrm{S}$ & $\mathrm{S}$ \\
\hline RGJU 11 & AG3 & S & S & S & S & S & S & S & S & S & $\mathrm{S}$ \\
\hline RGJG2 & AG5 & S & $\mathrm{S}$ & $\mathrm{S}$ & $\mathrm{S}$ & $\mathrm{S}$ & $\mathrm{S}$ & $\mathrm{S}$ & $\mathrm{S}$ & S & $\mathrm{S}$ \\
\hline RGJG4 & UD & $\mathrm{S}$ & S & $\mathrm{S}$ & $\mathrm{S}$ & $\mathrm{S}$ & $\mathrm{S}$ & $\mathrm{S}$ & $\mathrm{S}$ & $\mathrm{R}$ & $\mathrm{S}$ \\
\hline RGJW 15 & AG5 & S & S & $\mathrm{S}$ & S & S & S & S & S & S & S \\
\hline RHPF2 & UD & S & S & S & S & $\mathrm{S}$ & S & S & S & S & S \\
\hline RHRC 20 & AG2-3 & S & $\mathrm{S}$ & $\mathrm{S}$ & S & $\mathrm{S}$ & S & $\mathrm{S}$ & S & S & S \\
\hline RHRC 21 & AG4 & $\mathrm{S}$ & $\mathrm{S}$ & $\mathrm{S}$ & $\mathrm{S}$ & $\mathrm{S}$ & $\mathrm{S}$ & $\mathrm{S}$ & S & $\mathrm{S}$ & $\mathrm{S}$ \\
\hline RHRC 22 & AG2-3 & $\mathrm{S}$ & $\mathrm{S}$ & $\mathrm{S}$ & S & $\mathrm{S}$ & S & $\mathrm{S}$ & $\mathrm{S}$ & S & $\mathrm{S}$ \\
\hline RHRC 28 & AG3 & S & S & S & S & $\mathrm{S}$ & S & $\mathrm{S}$ & S & S & S \\
\hline RHRM3 & UD & S & S & S & S & S & S & S & S & S & S \\
\hline RHRM4 & AG2-2LP & $\mathrm{S}$ & $\mathrm{S}$ & $\mathrm{S}$ & $\mathrm{S}$ & $\mathrm{S}$ & $\mathrm{S}$ & $\mathrm{S}$ & $\mathrm{S}$ & $\mathrm{S}$ & $\mathrm{S}$ \\
\hline RHRG5 & AG2-3 & $\mathrm{S}$ & $\mathrm{S}$ & $\mathrm{S}$ & $\mathrm{S}$ & $\mathrm{S}$ & $\mathrm{S}$ & $\mathrm{S}$ & $\mathrm{S}$ & $\mathrm{S}$ & $\mathrm{S}$ \\
\hline RHRW16 & AG1 & S & S & S & S & S & S & S & S & S & S \\
\hline RHRW27 & UD & $\mathrm{S}$ & $\mathrm{S}$ & S & $\mathrm{S}$ & $\mathrm{S}$ & S & S & $\mathrm{S}$ & $\mathrm{S}$ & $\mathrm{S}$ \\
\hline RHRW32 & AG2-3 & S & $\mathrm{S}$ & $\mathrm{S}$ & S & $\mathrm{S}$ & S & S & S & S & $\mathrm{S}$ \\
\hline RJKM2 & AG2-3 & S & S & S & S & $\mathrm{S}$ & S & S & S & S & S \\
\hline RJKM8 & AG1 & $\mathrm{S}$ & $\mathrm{S}$ & $\mathrm{S}$ & $\mathrm{S}$ & $\mathrm{S}$ & $\mathrm{S}$ & $\mathrm{S}$ & $\mathrm{S}$ & $\mathrm{S}$ & $\mathrm{S}$ \\
\hline RJKM 15 & AG2-2LP & S & $\mathrm{S}$ & S & S & $\mathrm{S}$ & S & $\mathrm{S}$ & S & S & S \\
\hline RJKU 13 & AG5 & $\mathrm{S}$ & $\mathrm{S}$ & $\mathrm{S}$ & $\mathrm{S}$ & $\mathrm{S}$ & $\mathrm{S}$ & $\mathrm{S}$ & $\mathrm{S}$ & $\mathrm{S}$ & $\mathrm{S}$ \\
\hline
\end{tabular}




\begin{tabular}{|c|c|c|c|c|c|c|c|c|c|c|c|}
\hline RJHM1 & AG5 & $\mathrm{S}$ & $\mathrm{S}$ & $\mathrm{S}$ & $\mathrm{S}$ & $\mathrm{S}$ & $\mathrm{S}$ & $\mathrm{S}$ & $\mathrm{S}$ & $\mathrm{S}$ & $\mathrm{s}$ \\
\hline RJHU1 & AG1 & S & $\mathrm{S}$ & $\mathrm{S}$ & $\mathrm{S}$ & S & S & $\mathrm{S}$ & $\mathrm{S}$ & S & S \\
\hline RKLC1 & AG2-3 & $\mathrm{S}$ & $\mathrm{S}$ & $\mathrm{S}$ & $\mathrm{S}$ & S & $\mathrm{S}$ & $\mathrm{R}$ & $\mathrm{S}$ & S & $\mathrm{s}$ \\
\hline RKLC4 & UD & S & S & S & S & S & S & $\mathrm{R}$ & S & S & S \\
\hline RKNM8 & AG-3 & $\mathrm{S}$ & $\mathrm{S}$ & $\mathrm{S}$ & $\mathrm{S}$ & $\mathrm{S}$ & $\mathrm{S}$ & $\mathrm{S}$ & $\mathrm{S}$ & $\mathrm{S}$ & $\mathrm{S}$ \\
\hline RKNG9 & AG1 & S & S & S & S & S & S & S & S & S & S \\
\hline RMPM9 & AG3 & S & $\mathrm{S}$ & $\mathrm{S}$ & $\mathrm{S}$ & $\mathrm{S}$ & $\mathrm{S}$ & $\mathrm{S}$ & $\mathrm{S}$ & $\mathrm{S}$ & S \\
\hline RMPM10 & AG3 & S & S & $\mathrm{S}$ & $\mathrm{S}$ & $\mathrm{S}$ & $\mathrm{S}$ & $\mathrm{S}$ & $\mathrm{S}$ & $\mathrm{S}$ & $\mathrm{s}$ \\
\hline RMPM13 & AG2-3 & S & S & S & $\mathrm{S}$ & S & S & S & S & S & S \\
\hline RMPM23 & AG4 & S & S & S & $\mathrm{S}$ & S & S & S & S & S & S \\
\hline RMPG28 & AG2-3 & $\mathrm{S}$ & $\mathrm{S}$ & $\mathrm{S}$ & $\mathrm{S}$ & $\mathrm{S}$ & $\mathrm{S}$ & $\mathrm{S}$ & $\mathrm{S}$ & $\mathrm{S}$ & $\mathrm{S}$ \\
\hline RMPG29 & AG1 & S & S & S & S & S & S & $\mathrm{S}$ & S & S & S \\
\hline RMPP 30 & AG4 & $\mathrm{S}$ & $\mathrm{S}$ & $\mathrm{S}$ & $\mathrm{S}$ & S & $\mathrm{S}$ & $\mathrm{S}$ & S & S & $\mathrm{s}$ \\
\hline RMHM3 & AG2-3 & S & $\mathrm{S}$ & $\mathrm{S}$ & S & $\mathrm{S}$ & $\mathrm{S}$ & $\mathrm{R}$ & $\mathrm{S}$ & S & S \\
\hline RMHM6 & AG5 & S & S & S & S & S & $\mathrm{R}$ & S & S & S & S \\
\hline RMHG24 & AG1 & S & S & $\mathrm{S}$ & $\mathrm{S}$ & $\mathrm{R}$ & S & S & S & S & S \\
\hline RMHP21 & AG2-2 & S & S & S & S & S & S & S & S & S & S \\
\hline RORC9 & AG3 & S & S & $\mathrm{S}$ & $\mathrm{S}$ & S & S & S & S & S & $\mathrm{s}$ \\
\hline RPBC1 & AG3 & S & S & S & $\mathrm{S}$ & S & S & S & S & S & S \\
\hline RPBM 17 & AG4 & S & $\mathrm{S}$ & $\mathrm{S}$ & $\mathrm{S}$ & $\mathrm{S}$ & S & $\mathrm{S}$ & $\mathrm{S}$ & $\mathrm{S}$ & S \\
\hline RPBU5 & AG4 & S & $\mathrm{S}$ & $\mathrm{S}$ & $\mathrm{S}$ & S & S & $\mathrm{R}$ & S & S & $\mathrm{s}$ \\
\hline RPBU7 & AG3 & S & S & S & S & S & S & S & S & S & S \\
\hline RPBR 18 & AG5 & $\mathrm{S}$ & $\mathrm{S}$ & $\mathrm{S}$ & $\mathrm{S}$ & $\mathrm{S}$ & $\mathrm{S}$ & $\mathrm{S}$ & $\mathrm{S}$ & $\mathrm{S}$ & $\mathrm{S}$ \\
\hline RRJM7 & AG3 & S & S & S & S & S & S & S & S & S & S \\
\hline RRJG1 & AG5 & S & S & S & S & $\mathrm{S}$ & $\mathrm{S}$ & $\mathrm{S}$ & $\mathrm{S}$ & $\mathrm{S}$ & $\mathrm{s}$ \\
\hline RRJG3 & UD & S & $\mathrm{S}$ & $\mathrm{S}$ & $\mathrm{S}$ & $\mathrm{S}$ & S & $\mathrm{R}$ & $\mathrm{S}$ & S & S \\
\hline RTNU1 & AG1 & S & S & $\mathrm{S}$ & $\mathrm{S}$ & S & S & $\mathrm{S}$ & S & S & S \\
\hline RTNG5 & UD & S & S & S & $\mathrm{S}$ & S & S & S & S & S & S \\
\hline RUKM10 & AG2-2 & $\mathrm{S}$ & $\mathrm{S}$ & $\mathrm{S}$ & $\mathrm{S}$ & $\mathrm{S}$ & S & $\mathrm{S}$ & $\mathrm{S}$ & S & S \\
\hline RUKM8 & UD & S & S & S & $\mathrm{S}$ & S & S & S & S & S & S \\
\hline RUKU4 & AG3 & $\mathrm{S}$ & $\mathrm{S}$ & $\mathrm{S}$ & $\mathrm{S}$ & $\mathrm{S}$ & S & $\mathrm{S}$ & $\mathrm{S}$ & $\mathrm{S}$ & S \\
\hline RUPC 95 & UD & S & S & $\mathrm{S}$ & $\mathrm{S}$ & S & S & $\mathrm{S}$ & $\mathrm{S}$ & S & S \\
\hline RUPM42 & AG2-3 & S & S & S & S & S & S & S & S & S & S \\
\hline RUPM83 & AG1 & $\mathrm{S}$ & $\mathrm{S}$ & $\mathrm{S}$ & $\mathrm{S}$ & S & $\mathrm{S}$ & S & S & S & $\mathrm{s}$ \\
\hline RUPU 58 & AG2-2 & S & S & S & S & S & S & S & S & S & S \\
\hline RUPU 82 & AG1 & S & S & S & S & S & S & S & S & S & S \\
\hline RUPU 23 & AG3 & S & S & $\mathrm{S}$ & $\mathrm{S}$ & S & S & S & S & S & S \\
\hline RUPU 84 & AG2-2 & S & $\mathrm{S}$ & $\mathrm{S}$ & $\mathrm{S}$ & $\mathrm{S}$ & $\mathrm{S}$ & $\mathrm{S}$ & $\mathrm{S}$ & S & S \\
\hline RUPU 20 & AG2-3 & S & S & S & S & S & S & S & S & S & S \\
\hline RUPU 18 & AG2-3 & $\mathrm{S}$ & $\mathrm{S}$ & $\mathrm{S}$ & $\mathrm{S}$ & $\mathrm{S}$ & $\mathrm{S}$ & $\mathrm{S}$ & $\mathrm{S}$ & $\mathrm{S}$ & S \\
\hline RUPU 50 & AG2-3 & $\mathrm{S}$ & $\mathrm{S}$ & $\mathrm{S}$ & $\mathrm{S}$ & $\mathrm{S}$ & $\mathrm{S}$ & $\mathrm{S}$ & $\mathrm{S}$ & $\mathrm{S}$ & $\mathrm{S}$ \\
\hline RUPK8 & UD & S & $\mathrm{S}$ & $\mathrm{S}$ & $\mathrm{S}$ & S & S & $\mathrm{S}$ & S & S & S \\
\hline RUPG 103 & AG5 & $\mathrm{S}$ & $\mathrm{S}$ & $\mathrm{S}$ & $\mathrm{S}$ & $\mathrm{S}$ & $\mathrm{R}$ & $\mathrm{S}$ & $\mathrm{S}$ & $\mathrm{S}$ & $\mathrm{S}$ \\
\hline RUPG 106 & AG3 & S & S & S & $\mathrm{S}$ & S & $\mathrm{S}$ & $\mathrm{S}$ & $\mathrm{S}$ & $\mathrm{S}$ & $\mathrm{s}$ \\
\hline RUPP 93 & AG5 & S & $\mathrm{S}$ & $\mathrm{S}$ & $\mathrm{S}$ & $\mathrm{R}$ & S & $\mathrm{S}$ & S & S & S \\
\hline RUPL 104 & UD & S & S & S & S & S & S & S & S & S & S \\
\hline RUPM 80 & UD & S & $\mathrm{S}$ & $\mathrm{S}$ & $\mathrm{S}$ & S & S & $\mathrm{S}$ & $\mathrm{S}$ & $\mathrm{S}$ & S \\
\hline RS6 & AG1 & S & $\mathrm{S}$ & $\mathrm{S}$ & $\mathrm{S}$ & S & S & $\mathrm{S}$ & $\mathrm{S}$ & $\mathrm{S}$ & $\mathrm{s}$ \\
\hline RS7 & AG5 & S & S & S & S & S & S & S & S & S & S \\
\hline RS8 & AG4 & S & S & $\mathrm{S}$ & $\mathrm{S}$ & S & S & $\mathrm{S}$ & S & $\mathrm{S}$ & S \\
\hline RS9 & AG3 & S & S & S & S & S & $\mathrm{R}$ & S & S & S & S \\
\hline RS11 & AG2-2 & S & S & S & $\mathrm{S}$ & S & S & S & S & S & S \\
\hline RS15 & AG2-3 & S & S & S & $\mathrm{S}$ & S & S & $\mathrm{S}$ & S & S & S \\
\hline RS14 & AG2-2LP & S & S & S & S & S & S & S & S & $\mathrm{S}$ & S \\
\hline RJHC1 & AG1 & $\mathrm{S}$ & $\mathrm{S}$ & $\mathrm{S}$ & $\mathrm{S}$ & $\mathrm{S}$ & $\mathrm{S}$ & $\mathrm{S}$ & $\mathrm{S}$ & $\mathrm{S}$ & S \\
\hline RWBC1 & AG3 & $\mathrm{S}$ & $\mathrm{S}$ & $\mathrm{S}$ & $\mathrm{S}$ & S & S & S & S & S & S \\
\hline RWBC4 & AG5 & $\mathrm{S}$ & $\mathrm{S}$ & $\mathrm{S}$ & $\mathrm{S}$ & $\mathrm{S}$ & $\mathrm{S}$ & $\mathrm{S}$ & $\mathrm{S}$ & $\mathrm{S}$ & $\mathrm{S}$ \\
\hline
\end{tabular}

$\mathrm{R}=$ Resistant $(0-20 \%$ disease incidence $)$ and $\mathrm{S}=$ Susceptible $(>20-100 \%$ disease incidence $)$. 
Table 5. Pathogenic groups of $R$. solani and their differential cultivars

\begin{tabular}{cccc}
\hline Pathotype/group & $\begin{array}{c}\text { Cultivar } \\
\text { (Resistant) }\end{array}$ & $\begin{array}{c}\text { Isolate } \\
\text { (No.) }\end{array}$ & Isolate (AG) \\
\hline I & $\begin{array}{c}\text { KU 323 } \\
\text { (urdbean })\end{array}$ & 2 & RMHG24 (AG1) and RUPP93 (AG5) \\
II & $\begin{array}{c}\text { Uttara } \\
\text { (urdbean })\end{array}$ & 4 & RAPS3 (AG1), RMHM6 (AG5), RUPG103 (AG5) and \\
RS9 (AG3) \\
III & $\begin{array}{c}\text { KUG 216 } \\
\text { (urdbean) }\end{array}$ & 6 & RGJC18 (AG2-2), RKLC1 (AG2-3), RKLC 4 (UD), \\
IV & V 578 (cowpea) & 1 & $\begin{array}{c}\text { RMM3 (AG2-3), RPBS (AG4) } \\
\text { and RRJG3 (UD) }\end{array}$ \\
V & All 10 cultivars & 77 & RGJG 4 (UD) \\
\hline
\end{tabular}

\section{Discussion}

Rhizoctonia solani produced typical web blight /wet root rot symptoms on different pulse crops under diverse environmental conditions. The development of disease was favoured by wide range of atmospheric and soil temperature and relative humidity. Wide range of soil properties as $\mathrm{pH}, \mathrm{EC}$ and texture along with nutritional conditions as organic carbon, nitrogen and phosphorus also favoured disease development in the areas surveyed. Most of the areas surveyed showed low to medium level of soil organic carbon. Tewoldemedhin et al. (2006) reported variation in temperature requirement for the growth of $R$. solani and observed that the isolates of $R$. solani belonging to AG 2-2 and AG 4 HG-II had significantly higher optimum growth temperatures than those from other AGs. In the present study, the type of AGs were not correlated with either temperature or relative humidity requirement in the field conditions

The present findings clearly indicated that the disease is favoured by wide rage of soil ranging from silt, loam, sandy and clay. In general all type of soils was found suitable for the development of the disease in various crops. The pathogenicity of the combined as well as hybrid culture of different AGs showed that the first generation of hybrid culture of two AGs was more or less similar in the aggressiveness on susceptible cultivars of mungbean of their parental AGs.

First time through the present study, an attempts was made to standardize the differential cultivars for virulence analysis of the populations of $R$. solani originating from different pulse crops. Availability of resistant cultivars against the pathogen in pulse crops is major limitation for differentials. Therefore, a large number of cultivars of mungbean, urdbean and cowpea were evaluated against the pathogen with an aim to find out resistant cultivars to constitute an effective set of differentials. Out of 109 cultivars evaluated, ten cultivars having different degree of resistance and susceptibility against the pathogen ( 3 of mungbean, 4 of urdbean and 3 of cowpea) were selected to constitute a set of differentials for virulence analysis. The results confirmed that there were differences in virulence among the isolates tested. The results of virulence analysis of 90 isolates including 7 international isolates of AGs tester of $R$. solani indicated that most of the isolates caused susceptible reaction on the set of cultivars evaluated. The isolates were grouped into the five pathotypes and differential cultivars for each pathotype were determined. Attempts have not been made earlier to determine the pathotypes of $R$. solani. Scanty information is available on the pathogenic grouping of the limited number of the isolates on a few cultivars and the isolates were found variable in respect of pathogenicity. Similar to the present study Engelkes and Windels (1996) reported that the isolates of $R$. solani AG2-2IIIB and AG2-2 IV were pathogenic to sugarbeet and bean crops and the severity of the isolates on these crops differed. Bohlooli et al. (2006) observed that 81 isolates of $R$. solani isolated from roots and hypocotyls of bean showed variability in respect of virulence on the cultivars of beans. Tewoldemedhin et al. (2006) also observed differences among the virulence of $R$. solani isolates representing different AGs on different crops. The isolates of AG 2-2 and AG 4 HG-II were the most virulent on all crops evaluated. AG 2-1 was highly virulent on canola, moderately virulent on medic and lupin, weakly virulent on lucerne and barley, and nonpathogenic on wheat. AG-11 isolates were moderate to 
weakly virulent on all crops, with the exception of barley and wheat. AG-3 was weakly virulent on canola, lupin, and medic. In the present study, the isolates representing 7 AGs were highly variable on mungbean, urdbean and cowpea cultivars included in the present study, but there was no definite correlation in between AGs and cultivar or crops. Godoy-Lutz et al. (2008) observed at least six different subgroups of $R$ solani causing web blight symptoms in common bean. Khandaker et al. (2008) tested pathogenicity of two potato isolates of $R$. solani on 33 hosts including pulse crops and observed differences in the degree of pathogenicity of both the isolates in respect of hosts. Seventy eight isolates of $R$. solani were isolates from diseased lupin plants and various other crops in Alberta, Canada. Isolates belonging to AG-4 produced typical symptoms of stem rot and root rot on lupin seedlings and showed greater virulence compared with AG-2-1 and AG-2-2 isolates (Zhou et al., 2009). Caesar et al. (2010) observed significant differences among the isolates in respect of their virulence. Pathogenic behaviour of 368 isolates of $R$. solani representing 7 AGs collected from different leguminous crops from 16 agro-ecological regions of India were highly variable in pathogenicity and caused $10 \%$ to $100 \%$ wet root rot incidence in mungbean, urdbean and cowpea and $11 \%$ to100\% in chickpea (Dubey et al., 2011). The pathogenicity tests on Beta vulgaris revealed that $R$. solani AG2-2 IIIB and AG2-2 IV isolates were more virulent than $R$. cerealis (Taheri \& Tarighi, 2012).

\section{Conclusion}

Wet root rot/web blight caused by $R$. solani is an important disease of pulse crops. Pathotypes based on virulence on a set of differential cultivars is not known for the populations of $R$. solani. No attempt has been made in the past to develop a set of differential cultivars for pathotyping of the pathogen. Considering the wide host range of pulses, a set of differential cultivars consisting of mungbean, urdbean and cowpea was developed in the present study. Based on the virulence on the a set of differential cultivars, the populations of $R$. solani representing 7 AGs associated with pulse crops were grouped into 5 pathotypes and differential cultivar for each pathotype was identified. The weather and soil parameters like temperature, humidity, $\mathrm{pH}$, electric conductivity, soil texture, organic carbon, nitrogen and phosphorus influenced the disease development ranging from $2-48 \%$ in the areas surveyed for collection of the isolates. The pathotype groups generated in the present study were not corresponding to AGs/crop of origin/area of origin of the isolates. The information generated on pathotypes can be used for resistant breeding against the pathogen.

\section{Acknowledgement}

Authors are thankful to the Indian Council of Agricultural Research, New Delhi for providing financial support.

Divisional publication accession No. 6/12.

\section{References}

Agricultural Statistics at a Glance. (2010). Directorate of Economics and Statistics, Department of Agriculture, Ministery of Agriculture, Goverment of India.

Bohlooli, A., Okhovvat, S. M., \& Javan-Nikkhah, M. (2006). Pathogenicity of some Rhizoctonia solani isolates associated with root/collar rots on the cultivars of bean in greenhouse. Commun Agric. Appl. Biol. Sci., 71, 1197-1202.

Caesar, A. J., Caesar T., \& Maathuis, M. H. (2010). Pathogenicity, characterization and comparative virulence of Rhizoctonia spp. from insect-galled roots of Lepidium draba in Europe. Biol. Control, 52, 140-144. http://dx.doi.org/10.1016/j.biocontrol.2009.10.006

Carling, D. E. (1996). Grouping in Rhizoctonia solani by hyphal anastomosis. In Sneh, B., Jabaji-Hare, S., Neate, S., \& Dijst, G. (Eds), Rhizoctonia Species: Taxonomy, Molecular Biology, Ecology, Pathology, and Disease Control (pp. 37-47). Kluwer Academic Publishers, Dordrecht, The Netherlands.

Dubey, S. C. (2003). Integrated management of web blight of urd/mung bean by bio-seed treatment. Indian Phytopath., 56, 34-38.

Dubey, S. C., Bhavani, R., \& Singh, B. (2009). Development of Pusa 5SD for seed dressing and Pusa Biopellet 10G for soil application formulation of Trichoderma harzianum and their evaluation for integrated management of dry root rot of mungbean (Vigna radiata). Biol. Control, 50, 231-242. http://dx.doi.org/10.1016/j.biocontrol.2009.04.008

Dubey, S. C., Tripathi, A., Upadhyay, B. K., \& Thakur, M. (2011). Pathogenic behaviour of leguminous isolates of Rhizoctonia solani collected from different Indian agro-ecological regions. Indian J. agril. Sci., 81, 64-69.

Engelkes, C. A., \& Windels, C. E. (1996). Susceptibility of sugar beet and beans to Rhizoctonia solani AG-2-2 
IIIB and AG-2-2 IV. Plant Dis., 80, 1413-1417. http://dx.doi.org/10.1094/PD-80-1413

Godoy-Lutz, G., Kuninaga, S., Steadman, J., \& Powers, K. (2008). Phylogenetic analysis of Rhizoctonia solani subgroups associated with web blight symptoms on common bean based on ITS-5.8s rDNA. J. Gen. Plant Path., 74, 32-40. http://dx.doi.org/10.1007/s10327-007-0060-6

Kaiser, N. J. (1970). Rhizoctonia stem canker disease of mungbean in Iran. Plant Dis Rep., 54, 240-50.

Khandaker, M. M., Khair, A., \& Bhuiyan, M. K. A. (2008). Disease reaction of different crops against virulent potato isolates of Rhizoctonia solani Kühn. Bangladesh J. Bot., 37, 75-80. http://dx.doi.org/10.3329/bjb.v37i1.1567

Ogoshi, A. (1987). Ecology and pathogenicity of anastomosis and intraspecific groups of Rhizoctonia solani Kühn. Annual Rev. Phytopath., 25, 125-43. http://dx.doi.org/10.1146/annurev.phyto.25.1.125

Parmeter, J. R. Jr., \& Whiteny, H. S. (1970). Taxonomy and nomenclature of the imperfect state. In Parmeter, J. R. Jr., (Ed.), Rhizoctonia solani: Biology and Pathology (pp. 20-31). California press, Berkeley.

Taheri, P., \& Tarighi, S. (2012). Genetic and virulence analysis of Rhizoctonia spp. associated with sugar beet root and crown rot in the northeast region of Iran. Plant Dis., 96, 398-408. http://dx.doi.org/10.1094/PDIS-08-11-0661

Tewoldemedhin, Y. T., Lamprecht, S. C., McLeod, A., \& Mazzola, M. (2006). Characterization of Rhizoctonia spp. recovered from crop plants used in rotational cropping systems in the Western Cape province of South Africa. Plant Dis., 90, 1399-1406. http://dx.doi.org/10.1094/PD-90-1399

Zhou, Q., Chang, K., Hwang, S., Strelkov, S. E., Gossen, B. D., \& Chen, Y. (2009). Pathogenicity and genetic diversity of Rhizoctonia solani isolates from lupin and other crops in Alberta, Canada. Can. J. Plant Pathol., 31, 340-347. http://dx.doi.org/10.1080/07060660909507608 